\title{
A Reflective Approach to Assessing Student Performance in Cybersecurity Exercises .
}

\author{
Richard Weiss \\ The Evergreen State College \\ Olympia, WA 98505 U.S.A. \\ weissr@evergreen.edu
}

\author{
Michael E. Locasto \\ The University of Calgary \\ Calgary, Alberta, CA \\ locasto@ucalgary.ca
}

\author{
Jens Mache \\ Lewis \& Clark College \\ Portland, OR U.S.A. \\ jmache@lclark.edu
}

\begin{abstract}
Assessing student performance in cybersecurity labs and exercises is a difficult and time-consuming task. Simply recording the number of correct answers is inferior to in-depth assessment. Faculty are often best placed to offer expert feedback, advice, and guidance based on assessing student achievement and quality of performance for time-constrained exercises. Since this often takes place in the context of large classes and complex exercises, it can present obstacles to offering qualitative feedback. Yet, in some cases there is more information available that could simplify this task. This paper explores the use of command line history and visualization to add additional information and simplify the problem.
\end{abstract}

\section{Categories and Subject Descriptors}

C.2.0 [Computer-Communication Networks]: General-Security and Protection; K.3.2 [Computer and Information Science Education]: Computer science education

\section{General Terms}

Security, Measurement

\section{Keywords}

grading; assessment; cybersecurity; qualitative feedback; exercises; analysis skills; EDURange

\section{INTRODUCTION}

Because cyber security draws on so many subfields of computer science, it is inherently challenging for students to integrate this broad variety of knowledge, especially at the undergraduate level. Realistic cybersecurity exercises are not only difficult for students to master, but can also represent a significant amount of work for the instructor to adopt or design, test, and grade (i.e., assess). For

\footnotetext{
${ }^{*}$ This work was partially supported by NSF grants 1141341, 1141314,1516100 , and 156730
}

Permission to make digital or hard copies of all or part of this work for personal or classroom use is granted without fee provided that copies are not made or distributed for profit or commercial advantage and that copies bear this notice and the full citation on the first page. Copyrights for components of this work owned by others than ACM must be honored. Abstracting with credit is permitted. To copy otherwise, or republish, to post on servers or to redistribute to lists, requires prior specific permission and/or a fee. Request permissions from Permissions@acm.org.

SIGCSE '16, March 02 - 05, 2016, Memphis, TN, USA

Copyright is held by the authors. Publication rights is licensed to ACM. ACM 978-1-4503-3685-7/16/03 ...\$15.00.

http://dx.doi.org/10.1145/2839509.2844646 both network and systems security, the learning objectives span multiple levels and areas of concern. They include acquiring both conceptual understanding as well as skills in applying theory to practice. Hence there are a wide variety of questions for students to explore. As a way of illustrating such variety, we list some examples of such questions related to network security (similar ones exist for systems security). This list is by no means complete: it is merely intended to provide some context for the types of exploration and analysis a student might undertake.

- What is the topology and content of a target network? What are the best ways to perform surveillance and auditing? (This is the topic that we focus on in this paper.)

- What are the vulnerabilities of a system? Which ones are serious?

- Has the network been compromised? If so, when and how and how can it be restored to safer state that eliminates the vulnerability that led to the problem?

- Have network resources been exhausted? Can we tell the difference between legitimate traffic and malicious traffic?

- Has sensitive data been exfiltrated? If so, when, which data, and how?

Even this brief list begins to reveal that teaching and testing a student's understanding of the issues at play is no simple affair. It is not just a question of whether students found a correct answer by applying the tools, but how they found the answer. We believe that we will be able to provide better feedback to students and better assess their work if we incorporate additional information about what they did in the exercise rather than just a numerical score based on the number of correct answers. In cybersecurity exercises in particular, there may be multiple paths to a correct solution, and how a student (or team of students) proceeds along these paths may involve backing up and correcting false assumptions. As instructors and assessors of student performance, we are most interested in seeing evidence of this kind of growth in student knowledge and competence [2, 4].

In order to answer questions like these, students often need to analyze complex systems and data, and the answers are not always simple. For example, to figure out if a process is doing something anomalous, one might need to look through a trace with hundreds of system calls. This requires that one understand what type of system calls there are and what they do. Following this example, one would become familiar with strace and its options, as well as process forking and interprocess communication.

We want to assess multiple types of knowledge: conceptual understanding of what the problems are and what tools to use to solve 
them, skills in using tools, analytical reasoning to correctly and thoroughly interpret extensive output. Written exams are a limited tool for doing this. Even skills-based tests can produce misleading results. It is possible for a student to copy a solution from the Web without really understanding it. There are several frameworks for hands-on exercises that have the potential to provide additional information for assessing operational and analytical skills relevant to security. Several are discussed in Section 2. In this paper, we look at a specific example implemented in EDURange where we collected this type of data, and we share our observations about what information we were able to extract.

\section{RELATED WORK}

There are many frameworks that are designed to teach cyber security through hands-on exercises. SecKnitKit and SEED provide VMs that the students download and use to perform specific tasks. Timchenko et al. [12] created VMs that they install in their lab. As such, they do not provide an opportunity to collect information about the problem-solving process. Modifying traditional Capture the Flag competitions such as CSAW and iCTF are challenging and time-consuming tasks [3]. Competitions such as CCDC and Packetwars [5] have access to these data, but they require significant infrastructure and are not something one could use in the classroom.

There are several cloud-based virtual environments that could be modified to capture user interactions in the classroom. The RAVE provides each student with access to several hosts that are configured for the labs in Principles of Computer Security Lab Manual [8]. NICE-challenges provides a more extensive environment with a collection of open-ended challenges that focus on securityrelated IT skills [7]. There are some exercises implemented in DETERlab [9]. EDURange provides a virtual environment in a public cloud with about six exercises, several of which have questions for students to answer [13].

Most of the computer science literature on assessment of learning seems to address programming and computational thinking. While this is a very important part of Computer Science, it does not address many of the aspects of security education. Many of the tasks that a penetration tester must perform involve understanding network protocols, and behaviors of operating systems and database management systems. Of course, there are issues related to secure coding practices and reverse engineering of executable code that require a solid understanding of programming concepts. Furthermore, there is great value in teaching students not to make simple coding mistakes [11]. Nevertheless, some researchers have tried to emphasize that programming is not just the ability to generate code; it may also be regarded as a way of thinking, decomposing, and solving problems ("Computing Curricula 2001", Marion et al [6]). As such, collecting data other than the final product, i.e. the program, is important for providing feedback to students.

The work on learning programming concepts that has inspired us has been Soloway [10]. This work assessed students learning to program on whether they learned the fundamental concepts. More recently, researchers have started to apply machine learning techniques to compare students to each other as a way of recognizing groups of students who are missing some of the concepts [1]. This approach has the advantage of being scalable. In that work, the researchers instrumented the Eclipse IDE so that every time the student compiled a file, it would save the list of API calls, the abstract syntax tree (AST), and the frequencies of key words. These features were used to compute the distances between any two programs. In addition to being able to predict exam scores, their pedagogical insight was that the development path contains more information than the resulting program.

\section{THE EXERCISE}

The exercise that we studied is a seemingly simple one of network reconnaissance. It asks students to determine the number of live hosts (if any) along with any running network services on those hosts in a network (different from their own) with roughly $2^{15}$ IP addresses. Students have an hour to complete the task, and it could take all of that time if not done efficiently. Each "path" that a student takes is a sequence of activities where the student executes programs to interact with the scenario environment. We record this path by capturing the Linux bash history of commands that the student enters.

There are also a few different levels for this exercise, depending on how much the students know about nmap. For example, in the simplest case, all of the hosts respond to ping, which can be used to do an efficient scan. At more difficult levels, some hosts may not respond to ping or even have open TCP ports. When the student is scanning for open ports, there could be a firewall that is blocking responses.

This exercise captures some key abilities, such as finding strategies for limiting the amount of data to be searched or finding faster, parallel ways of searching it. It also illustrates one of the ways in which cybersecurity relates to many specialties within computer science. This exercise could be used in a Networking class or Security class to (1) review basic network protocols (2) discuss topics such as netmasks, routing, IP addresses, (3) introduce scanning as "listening" to the sequences of packets that nmap produces in response to different requested scan types (4) discuss high-level strategies for efficient and/or quiet scanning.

\section{DATA COLLECTION}

For the reconnaissance exercise, we collected the bash histories from two classes at two different schools. There were a total of 24 teams of students. Each line was tagged with the primary command. Some commands were identified as significant, e.g. nmap was the primary command that we were looking for, and we identified that as significant. We also distinguish among different instances of significant commands by looking at the options that were used, e.g. nmap $-\mathrm{sn}-\mathrm{n}$. The sequence of commands that are typed by the student form a long list, which is generally difficult to interpret structurally. For purposes of visualization, we collapse commands that are similar, i.e. have similar options and arguments, into a single node in a directed graph. Although some temporal information is potentially lost, this gives a representation that can visually decompose the graph into chains and cycles that correspond to phases in the students' path, as can be seen in Figure 1. We also include some other commands such as ls or grep which are not specific to the task, but give potential information about what the student might be trying to do. One can decompose the graph into roughly four subgraphs. Sometimes a subgraph will correspond to a particular task or phase, such as finding all hosts or scanning ports on specific hosts. We did this for the teams and looked for patterns and similarities between the graphs.

\section{RESULTS AND DISCUSSION}

For each team, we analyzed the graphs of the nmap options that each team used and a qualitative summary of their activity along with a quantitative assessment of their scanning results (i.e., whether and to what extent they identified the correct target IP addresses and service ports). We then reflect on the difference between these two methods of assessing the quality and correctness of student performance in the context of a cybersecurity exercise.

Section 3 describes the exercise that faced each team. Although 
this exercise has the practical effect of teaching nmap-specific scanning knowledge, its primary formulation has the underlying goal (i.e., learning objective) of providing students with an opportunity to develop their analysis skills. In this case, the exercise presents students with a twofold opportunity to (1) plan their approach to scanning a large network (which has a very real time cost) and (2) understand what each of their expressed scans (via the command line parameters) is actually asking of the network based on the actual packet types or content used by nmap to initiate and execute the scan. Thus, the purpose of the exercise is to confront the student with these more sophisticated issues in addition to learning nmap "lingo."

Thus, in our assessment approach, we seek to comment on the teams' abilities to both learn about nmap's use and to use it effectively and efficiently (thereby demonstrating that they understand the tool's operation and effect on the network). This latter point is a crucial piece of our intended feedback to the student. Through the very design of the exercise and its inherent dependence on the significant amount of time needed to scan a large and mostly empty IP range, we are seeking to demonstrate to the student that they need to acquire an understanding of how the tool works, not merely the ways it is invoked in standard examples. The professional and pedagogical lesson we wish to drive home is that Computer Science education involves creativity, analysis, and problem solving - not the brute-force regurgitation of examples copied from the Web.

We designed the exercise to do this more effectively than any well-intentioned words of caution and warning, and our assessment will seek to point out and reinforce this lesson to the teams. It is precisely because of the subtle nature of this learning objective that we must (as instructors) engage in a more detailed study and evaluation of the students work - not just whether they "got the right answer" by returning a list of IP addresses. Hence, the need to provide better, more meaningful and professionally relevant feedback drives our need to approach assessment of these types of tasks with a more detailed analysis. In short, if we wish to raise the level of our students' learning, we have to raise the quality of our own assessment. Creating better learning outcomes does not simply rest on giving students more assignments, or more cleverly constructed assignments, but also in providing them with better constructed assessment of their performance. In the next subsection, we summarize the information we gained from the traces for some of the teams.

\subsection{Qualitative Assessment}

\section{Team 1 .}

In one of the longest traces of the data set, this team begins by scanning a smaller subnet (the 10.0.0.0/18), perhaps realizing that the original task may take some time, and they place a bet that most hosts might reside in the 'beginning' or lower region of the $/ 17$ space. They then quickly switch to fairly aggressive ping scans of the entire /17. They find a handful of hosts, and attempt to ping and nmap these hosts (along with their own host and the router). This team does not ever seem to reach the full answer, but they do exhibit quite an appetite for nmap and seem to do a satisfactory job of exploring a range of the tool's options.

\section{Team 2.}

In an example of a brief scanning episode, Team 2 consults the nmap manual page and then a fairly aggressive ping scan of the entire /17. They also use the $-\mathrm{n}$ parameter to avoid DNS resolutions. They seem to find the host 10.0.0.6 early in the address space, concluding their very short trace ( 7 invocations of nmap in total) by aggressively default scanning this host. A somewhat mediocre attempt that stops at the first hint of reply from the simulated network.

\section{Team 3.}

Team 3's record is also fairly brief, but they show several noteworthy characteristics. They begin by applying nmap to their own host and consulting the manual documentation about nmap. This shows a good amount of willingness to explore the use of the tool before diving into the scan headlong. They initially use a default nmap scan of the entire /17; perhaps realizing this is taking too long, they attempt to switch to more verbose output and a scan that will report the OS type (a tempting option that is a distraction at this point in the game). They then seem to discover the '- $T$ ' parameter and execute increasingly aggressive scans. This is a good example of a team that improves, but in which a traditional approach to assessment would treat very harshly indeed. They demonstrated more progress than Team 2, even though they did not find any hosts In the graph we see a loop that includes reading the man pages and then invoking nmap again.

\section{Team 4.}

In the course of a medium-length trace, Team 4 manages to ping scan the entire /17 and move quickly on to aggressively (i.e., with the -T4 and -A options) scanning a set of individual IP addresses. They clearly solved the problem and understood the tool. The graph is much smaller than that for Team 1, but the complexity is hidden in the loops of length 1.

\section{Team 5}

Team 5's trace amounts to a relatively small amount of work; they initiate a brute-force default scan of the entire /17, eventually redirecting output to a file. They spend the rest of the trace checking the status of this file with the ls command. Not much evidence of results or learning about the tool's operation. A relatively poor performance which we cannot yet chalk up to either ignorance of or over-confidence in the tool, although the unsophisticated use of nmap (i.e., default scan type, no use of $-n$ ) argues for the former case. The graph has some similarities to that of Team 4, except that the loop does not contain any of the significant commands.

\section{Team 6.}

Team 6 is a good example of a team that doesn't seem to get anywhere in terms of assembling a viable list of 'results', but they do spend a fair amount of time experimenting with and evolving the list of command line switches and parameters they pass to nmap.

\section{Team 7.}

One of the longer traces, team 7 also shows signs of modest command line expertise by starting scans in the background and redirecting output to files, occasionally checking the progress of this output. This team also consults the nmap documentation before initiating any scans. They begin with a brute-force scan of the /17 space; this scan eventually yields fruit, and they go on to scan individual machines. This team also seems to get deep into the task, because they end with attempts to scan for web servers (port 80) in the entire net and then attempt to figure out what a service running on a non-standard port on one of the hosts is. From the activity in this trace, this team looks (a) quite competent (if ultimately uninspired and sub-optimal) and (b) able to achieve the answer and explore some of the intended (i.e., purposefully configured) 'weirdness' in the simulated network. 


\section{Team 10.}

Team 10 (graph not shown) does a large number of fast scans (-T5), each with slightly different options. They seemed to be preoccupied with specifying port ranges. This is a distraction because nmap does a good enough job at finding hosts using just a small number of ports. An explanation for why they did not find any hosts is probably that they did not wait for any of their scans to complete.

\subsection{Recommendations}

Assessing student work, particularly for certain kinds of interactive security exercises and labs, can be an onerous task for an instructor or TA that wishes to provide meaningful and in-depth feedback. Our experience turning student command sequences into graphs and writing up our observations leads us to see several ways in which these artifacts can be used in the classroom and for a variety of feedback.

1. Use as peer feedback. Get students and teams to exchange their own traces and talk through them with team members or other teams. Such peer feedback can be a low stress way of sharing experiences and results across teams without the pressure of being graded by an instructor or TA.

2. Use as a class review artifact. Anonymized traces and graphs can be used to spur whole-class discussion; each trace can be displayed and the pros and cons of the team's approach discussed by the entire class or lab section. This is similar to many code review activities.

3. Use as contextualized feedback. Getting students to improve their performance often requires them to understand where they went wrong and what alternatives could have led to better performance. Grading rubrics and score breakdowns often only communicate the relative weightings of different parts of assignments. In our approach, the graph augments the scoring rubric and individual feedback. Each student or team receives the annotated graph; the graph provides context and meaning to the rubric for the lab or assignment and a basis for interpreting the written feedback from the instructor and for self-reflection.

4. Use as an instructor planning tool. How students perform in clusters or groups can help inform the instructor where additional review or further reinforcement is needed; the graphs can show where common mistakes are made, or where students might have missed possible solutions to the problem at hand. In performing a reflective assessment of the exercise and attempting to explain what each team was doing, it often became apparent what each team's mental state might have been, and what information or insight they may have missed. This kind of reflection can lead the instructor to have some insight about hints or resources or unforeseen obstacles due to the design of the exercise.

5. Use as a "live" scoring method for competitions. Capturethe-flag style competitions are popular cybersecurity exercises. Such environments often benefit from a scoreboard, but showing (at least to the instructor) a graphical summary of each team's progress can help the instructor provide rapid feedback during the exercise itself. The utility of this approach is greatly enhanced if it can be largely automated. We note that the obvious analog here in programming courses is to an instructor reading and hand-grading hundreds or thousands of lines of code of a student program rather than just comparing output or running a few test cases. Such an approach is not scalable, and we are looking for ways to lighten the manual burden of this informal modeling of sequences of student behavior. Fortunately, the type of data we study is highly amenable to scripting and automated display.

\subsection{Alternatives}

One could suggest that a written exam could easily extract this type of information. One can design open-ended exams, but these are often very difficult and time-consuming to grade, especially for large classes or with little TA help. On the other hand, while they are easy to grade, there are problems with short-answer questions. In the case of network reconnaissance, we want to avoid asking students to memorize a set of commands that faculty deem as good a priori. We want to capture the process that students go through to find the answer because that is indicative of their ability to solve novel problems.

\section{CONCLUSION}

The key result that we have shown is that there is useful information in the bash history of students' commands that is not contained in the artifact that results from the completion of the set of tasks that was assigned. Teams 1, 4, and 7 did very well, but they used somewhat different approaches. Teams 3 and 6 did not find the answers, but met some of the learning goals. We did see one team that miraculously knew the IP addresses of the target hosts without seeming to scan the network. For this network reconnaissance exercise, whether or not students produced the correct answers, i.e. the correct IP addresses, ports and services, would not have been an accurate assessment of their understanding, e.g.,

1. By copying examples from the man pages or searching the Web, students could reproduce the right commands and options that will yield correct results.

2. Students could be on the right track to finding a good solution but run out of time or be missing just one piece of the solution.

3. Students may have understood the concepts but not have mastered all of the skills.

A significant amount of this information can be recovered from the history of what the students actually did in the course of their investigation. In all, this kind of informal modeling approach was practically useful for this exercise and generating meaningful feedback for the students.

\section{Acknowledgments}

We would like to thank Franklyn Turbak and Brian Fite for many suggestions and conversations about EDURange. We thank our students, especially Kahea Hendrickson, Isaak Yansane-Sisk, and Jeff Ladish. We also thank the reviewers for their useful and extensive comments.

\section{REFERENCES}

[1] Blikstein, P., Worsley, M., Piech, C., Sahami, M., COOPER, S., AND Koller, D. Programming pluralism: Using learning analytics to detect patterns in the learning of computer programming. Journal of the Learning Sciences 23, 4 (2014), 561-599.

[2] Cooper, S., Nickell, C., Piotrowski, V., Oldfield, B., Abdallah, A., Bishop, M., Caelli, B., Dark, M., Hawthorne, E. K., Hoffman, L., Pérez, L. C., Pfleeger, C., Raines, R., Schou, C., And BRYNIELSSON, J. An exploration of the current state of 
information assurance education. SIGCSE Bull. 41, 4 (Jan. 2010), 109-125.

[3] Doupé, A., Egele, M., Caillat, B., Stringhini, G., YAKin, G., ZAND, A., CAVEdon, L., AND Vigna, G. Hit'em where it hurts: a live security exercise on cyber situational awareness. In Proceedings of the 27th Annual Computer Security Applications Conference (2011), ACM, pp. 51-61.

[4] Endicott-Popovsky, B. E., And Popovsky, V. M. Application of pedagogical fundamentals for the holistic development of cybersecurity professionals. ACM Inroads 5, 1 (Mar. 2014), 57-68.

[5] Fite, B. The PacketWars Competition, 2015.

[6] Marion, B., Impagliazzo, J., St Clair, C., Soroka, B., AND WHITFIELD, D. Assessing computer science programs: what have we learned. ACM SIGCSE Bulletin 39, 1 (2007), 131-132.

[7] NeSTLER, V. NICE challenge project, 2015.

[8] Nestler, V. Principles of Computer Security Lab Manual, 4 ed. McGraw-Hill Education, 2015.

[9] Peterson, P. A. H., And Reiher, P. L. Security exercises for the online classroom with deter. In Proceedings of the 3rd International Conference on Cyber Security Experimentation and Test (Berkeley, CA, USA, 2010), CSET'10, USENIX Association, pp. 1-8.

[10] Soloway, E., AND Ehrlich, K. Empirical studies of programming knowledge. Software Engineering, IEEE Transactions on SE-10, 5 (Sept 1984), 595-609.

[11] TAYLOR, B., AND KaZA, S. Security injections: Modules to help students remember, understand, and apply secure coding techniques. In Proceedings of the 16th Annual Joint Conference on Innovation and Technology in Computer Science Education (New York, NY, USA, 2011), ITiCSE '11, ACM, pp. 3-7.

[12] Timchenko, M., And Starobinski, D. A simple laboratory environment for real-world offensive security education. In Proceedings of the 46th ACM Technical Symposium on Computer Science Education (2015), ACM, pp. 657-662.

[13] Weiss, R. S., Boesen, S., Sullivan, J. F., Locasto, M. E., MAChE, J., AND NILSEN, E. Teaching cybersecurity analysis skills in the cloud. In Proceedings of the 46th ACM Technical Symposium on Computer Science Education (New York, NY, USA, 2015), SIGCSE '15, ACM, pp. 332-337.

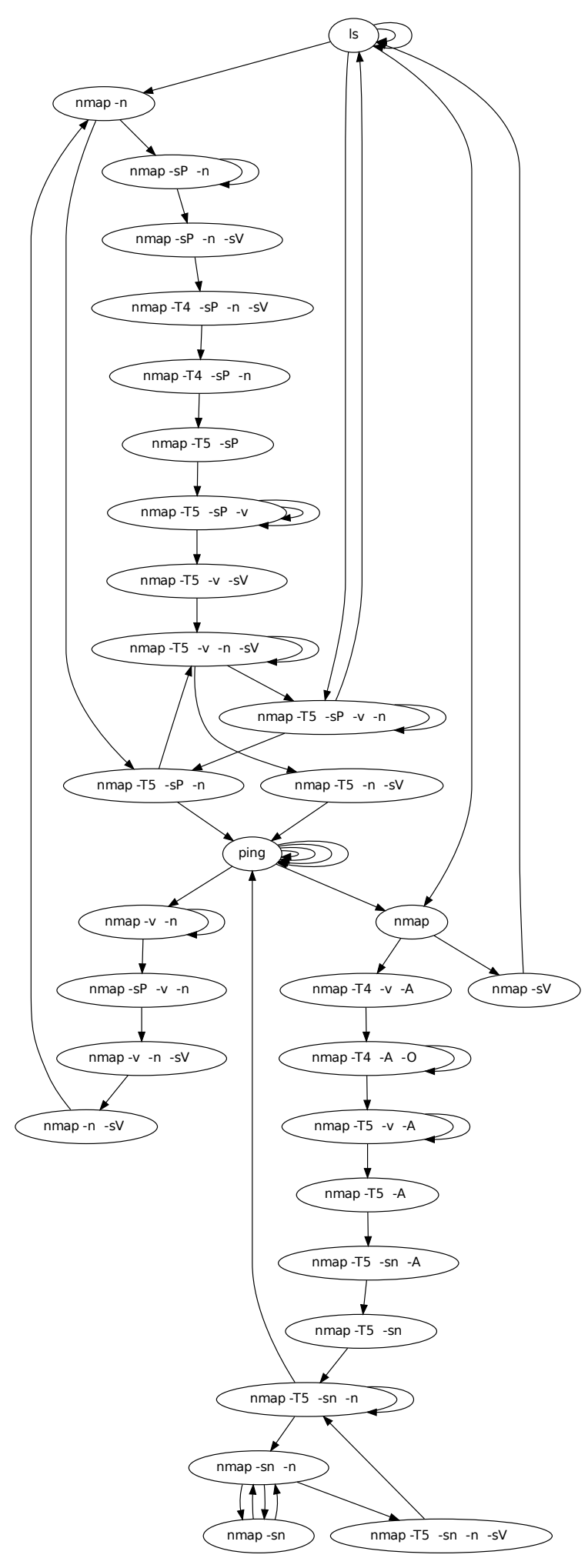

Figure 1: A directed graph of students' path 


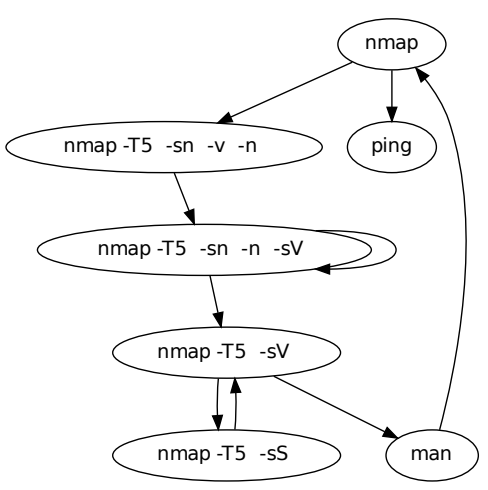

Figure 2: Team 2

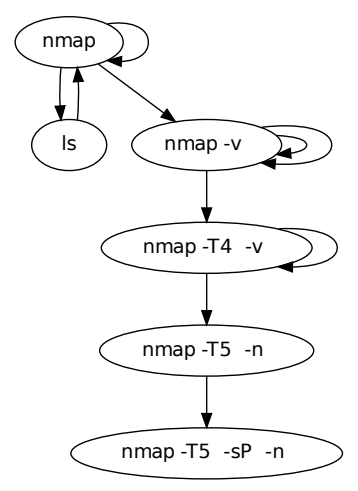

Figure 3: Team 3

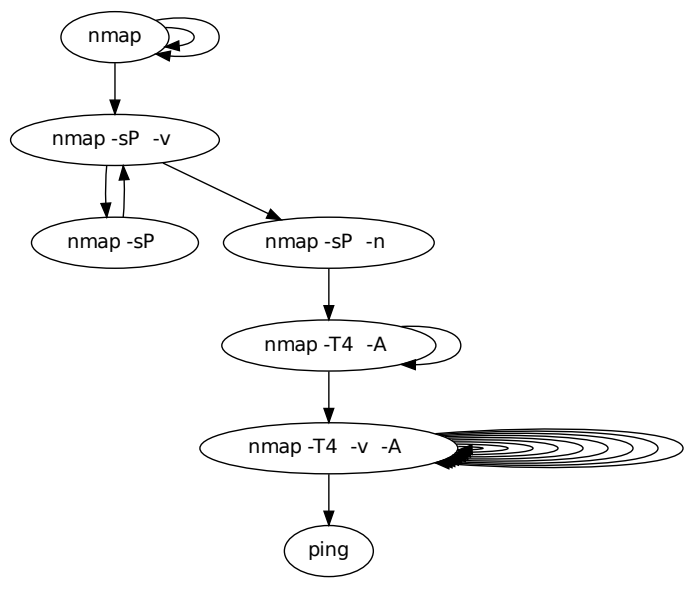

Figure 4: Team 4

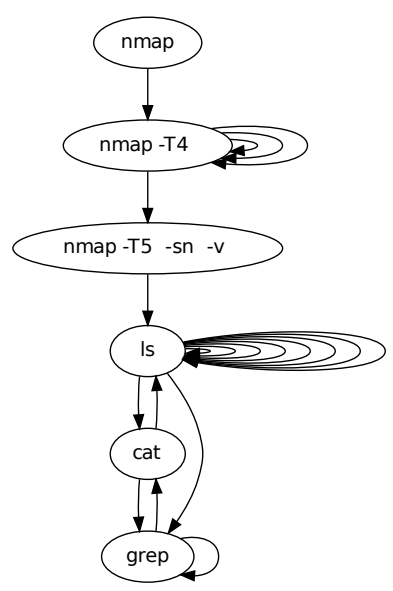

Figure 5: Team 5

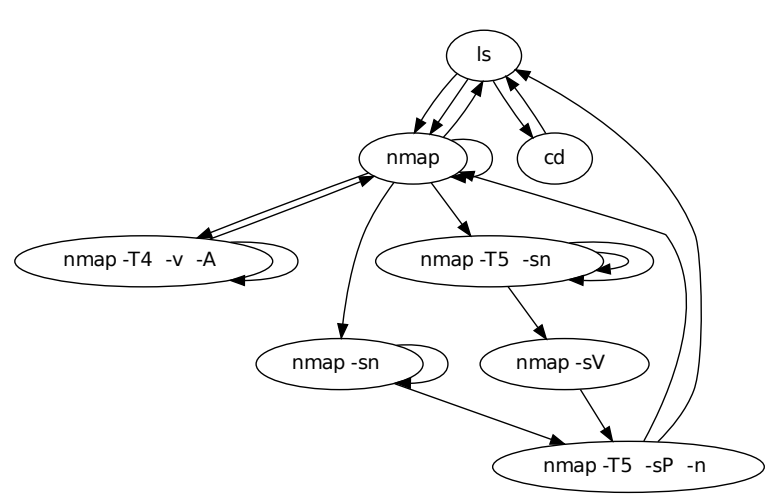

Figure 6: Team 6

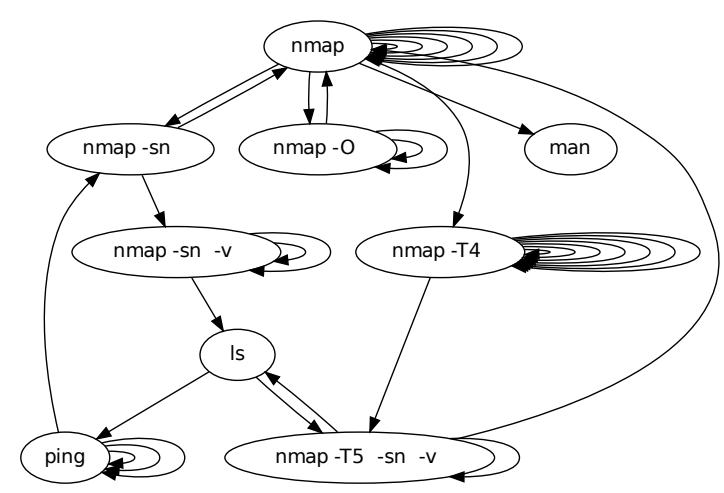

Figure 7: Team 7 